\title{
In vivo proton magnetic resonance spectroscopy of breast cancer: a review of the literature
}

\author{
Jonathan KP Begley', Thomas W Redpath², Patrick J Bolan ${ }^{3}$ and Fiona J Gilbert*
}

\begin{abstract}
An emerging clinical modality called proton magnetic resonance spectroscopy ('H-MRS) enables the noninvasive in vivo assessment of tissue metabolism and is demonstrating applications in improving the specificity of MR breast lesion diagnosis and monitoring tumour responsiveness to neoadjuvant chemotherapies. Variations in the concentration of choline-based cellular metabolites, detectable with 'H-MRS, have shown an association with malignant transformation of tissue in in vivo and in vitro studies. ${ }^{1} \mathrm{H}$-MRS exists as an adjunct to the current routine clinical breast MR examination. This review serves as an introduction to the field of breast ${ }^{1} \mathrm{H}-\mathrm{MRS}$, discusses modern high-field strength and quantitative approaches and technical considerations, and reviews the literature with respect to the application of ${ }^{1} \mathrm{H}-\mathrm{MRS}$ for breast cancer.
\end{abstract}

\section{Introduction}

Breast cancer remains a significant cause of morbidity and mortality in women internationally [1]. Proton magnetic resonance spectroscopy $\left({ }^{1} \mathrm{H}-\mathrm{MRS}\right)$ represents a non-invasive and non-ionising method of characterising a user-selected volume of tissue on the basis of the metabolic (chemical) content. ${ }^{1} \mathrm{H}$-MRS has been evaluated as an adjunct modality to breast MRI for increasing the specificity in differentiating malignant from benign tumours, in addition to its potential in monitoring responsiveness to chemotherapy.

This article aims to review choline related ${ }^{1} \mathrm{H}$-MRS in breast assessment, and to discuss the qualitative and quantitative approaches to ${ }^{1} \mathrm{H}$-MRS in breast cancer investigation. Both approaches to the technique will be described, along with a summary of the reported studies.

*Correspondence: fjg28@medschl.cam.ac.uk

${ }^{4}$ University of Cambridge, Radiology Department, Addenbrooke's Hospital,

Cambridge CB2 OQQ, UK

Full list of author information is available at the end of the article

\section{Methods}

English language studies investigating single-voxel (SV) or multi-voxel evaluation of the total choline containing resonance using in vivo ${ }^{1} \mathrm{H}$-MRS assessment of preoperative local or advanced breast cancer in human adult females, with or without additional assessment of benign or normal breast tissues, were sought. MEDLINE and EMBASE were searched for the period January 1990 to February 2012 using English language restrictions. The reference lists of relevant prior reviews were examined. Authors were contacted to identify relevant additional studies. Only full peer-reviewed articles were included. Sensitivity and specificity values in this review are expressed in terms of the percentage of correctly diagnosed malignant and benign lesions, respectively.

\section{Breast cancer physiology and ${ }^{1} \mathrm{H}-\mathrm{MRS}$}

${ }^{1} \mathrm{H}$-MRS assessment of the human breast demonstrates a number of distinct resonances attributable to choline, glycerides (esters of fatty acids and glycerols), saturated and unsaturated fatty acid, and water [2]. Numerous breast ${ }^{1} \mathrm{H}$-MRS studies performed in vivo have reported the association of a resonance at approximately 3.2 parts per million (ppm) with malignancy [2-8]. High-resolution high-field strength ex vivo analysis has revealed that a number of chemical compounds contribute to this single peak, including major contributions from free choline (3.19 ppm), phosphocholine (3.21 ppm), and glycerophosphocholine (3.22 ppm), as well as minor contributions from phosphoethanolamine (3.23 ppm), glucose (3.26 ppm), taurine (3.25 ppm), and myoinositol (3.27 ppm) [9]. With in vivo analysis these peaks undergo linebroadening and superposition and are generally represented by a single combined resonance at clinical field strengths as high as 4 Tesla $(\mathrm{T})$. Due to the major contribution of choline-containing metabolites to the composite signal at $3.2 \mathrm{ppm}$, it is commonly referred to as the total choline containing resonance (tCho). Figure 1 demonstrates a breast cancer on MRI and the corresponding $\mathrm{SV}{ }^{1} \mathrm{H}-\mathrm{MRS}$ spectrum acquired.

The relative concentrations of certain cellular metabolites change when cells transform from the normal state to a malignant form. Specifically, metabolic variations 


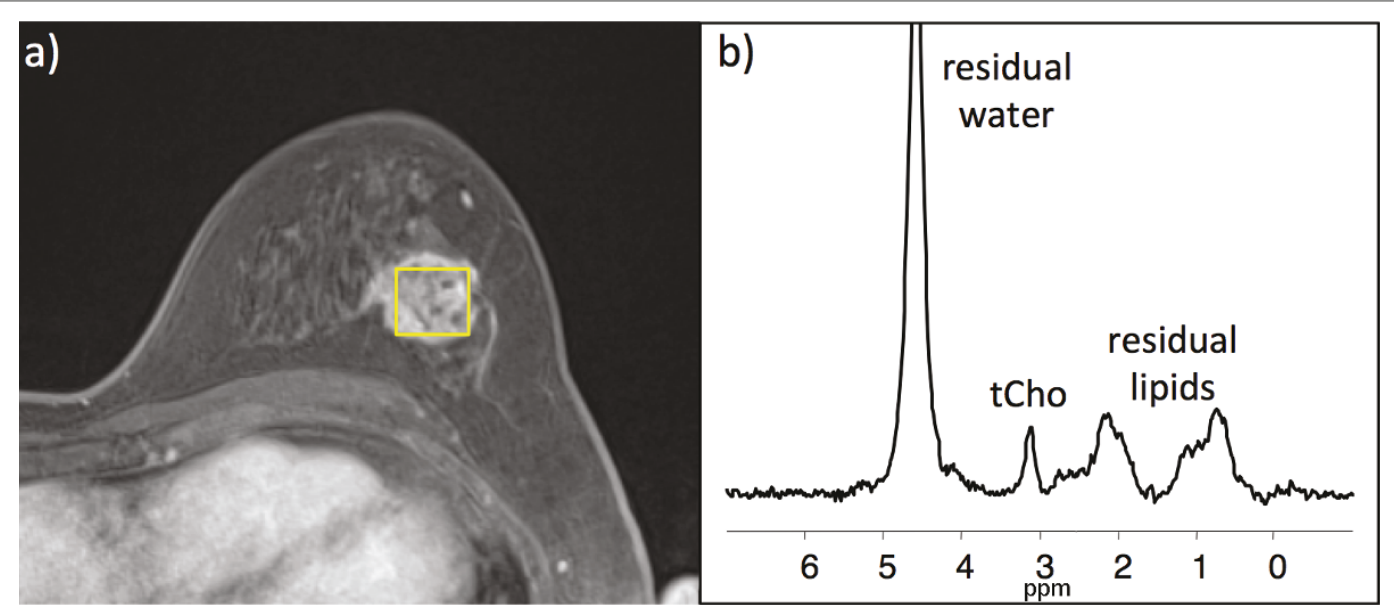

Figure 1. (a) A post-contrast gradient echo (TR/TE = 4.4/1.1 ms) 1.5 T MRI from a 51-year-old woman with invasive ductal carcinoma demonstrating single-voxel ${ }^{1} \mathrm{H}$ magnetic resonance spectroscopy ('H-MRS) voxel positioning. (b) The corresponding water and lipid suppressed single-voxel ${ }^{1} \mathrm{H}-\mathrm{MRS}$ spectrum acquired from the voxel, indicating the resonances present. Spectroscopy parameters: PRESS single voxel $15 \times 17 \times 20 \mathrm{~mm}$, TR/ $\mathrm{TE}=3,000 / 125 \mathrm{~ms}, 128$ averages, CHESS water suppression, MEGA/BASING lipid suppression. ppm, parts per million; tCho, total choline-containing resonance.

in the cell membrane resulting from proliferation, principally involving phosphocholine, manifest as variations in the concentration of choline-containing molecules. The tCho amplitude as determined by in vitro ${ }^{1} \mathrm{H}$ MRS has shown positive correlation with the metabolic proliferative activity of malignant cells [10]. In vivo investigation has reported that at least an order of magnitude more phosphocholine is present in breast cancer cells than normal mammary epithelial cells [11]. However, two in vitro studies $[12,13]$ show that cholinecontaining metabolite concentrations remain low in cultured normal human mammary epithelial cells when they proliferate at a similar rate to tumour-derived lines. This suggests that proliferative changes alone cannot completely account for the elevated choline metabolic activity seen in tumours.

\section{Single-voxel spectroscopy}

$\mathrm{SV}{ }^{1} \mathrm{H}-\mathrm{MRS}$ is considered the most suitable method for the assessment of distinct individual lesions. Adequate shimming has been shown to be more feasible with this approach. A major disadvantage with SV versus multivoxel spectroscopy is that SV acquisitions permit only a single lesion to be evaluated at a time. In addition, there is the inherent inability of SV methods to demonstrate spatial variability in biochemistry over a large area of heterogeneous tissue [14]. SV spectroscopy also requires lesion location to be known for accurate voxel placement; therefore, spectroscopic sequences should follow contrast injection and require a radiologist or technician to decide on appropriate voxel placement before ${ }^{1} \mathrm{H}$-MRS sequences.

\section{Multi-voxel spectroscopy}

Known either as chemical shift imaging or magnetic resonance spectroscopic imaging (MRSI), this technique enables the simultaneous acquisition of a grid of multiple spectroscopic voxels using gradient encoding. A major benefit of this method is the capacity to acquire a matrix of multiple spectra within a slice of anatomical structure such as the brain or breast, thereby enabling 'mapping' of spatial variations of in vivo metabolites. This may be advantageous in demonstrating cancer infiltration or margins. The capability to evaluate multiple lesions simultaneously is significant given that dynamic contrast enhanced MRI (DCE-MRI), which is generally performed immediately prior to ${ }^{1} \mathrm{H}-\mathrm{MRS}$, can demonstrate previously unknown additional lesions. The greater spatial coverage volume afforded by MRSI, as well as the ability to retrospectively shift the voxel grid to better align with a lesion of interest, makes it a more amenable technique for pre-contrast ${ }^{1} \mathrm{H}$-MRS acquisitions where the lesion location is uncertain. MRSI also makes it possible to simultaneously assess normal breast parenchymal tissue (perhaps with the potential for referencing). In practice, however, several issues present significant challenges to the installation of MRSI. One of the most important such factors is the difficulty associated with achieving adequate shimming over such a large volume of tissue, to the extent required for robust simultaneous spectroscopic acquisitions from each voxel within the matrix [14]. The spatial localisation is typically not as precise as in SV, which increases partial volume errors. Furthermore, the quantitative analysis approaches (described below) are more difficult to implement for MRSI than SV 
due to greater receive coil variation and the time needed to acquire an internal water reference spectrum.

Several breast MRSI studies have been conducted to date [15-22]. Potential implementations of the technique are highlighted with the following two publications. A 20081.5 T MRSI study by $\mathrm{Hu}$ et al. [15] involving ten women with known or suspected breast cancer reported the use of a tCho signal-based colour-coded map of a breast lesion to detect an intra-lesion choline 'hot spot'. Such a demonstration highlights the potential for MRSI to be used as a method for selecting the optimal site for breast biopsy.

A 20061.5 T MRSI study by Su et al. [16] involving 14 breast cancer patients investigated the correlation between tCho signal-to-noise ratio (SNR) and DCE-MRI vascular attributes. They reported a significant linear correlation of tCho SNR with the percentage of enhancement two minutes following contrast administration $(P=0.002)$, as well as with the pharmacokinetic properties $\mathrm{K}^{\text {trans }}$ (dynamic contrast enhanced derived transfer coefficient; $P=0.003$ ) and $\mathrm{K}_{\mathrm{ep}}$ (exchange rate constant; $P=0.002$ ). These results suggest that there is a relationship between choline metabolic activity and angiogenic activity. As choline is involved in cellular proliferation, it is logical that angiogenesis increases to support tumour metabolic requirements.

\section{Qualitative approach to tCho}

Roebuck et al. [3] suggested the potential of utilising tCho as a biomarker of breast malignancy in 1998. The qualitative approach to tCho, first reported for use in in vivo breast assessment by this team, involves the subjective determination by an observer as to whether a distinct resonance at approximately $3.2 \mathrm{ppm}$ is present. No objective statistical analysis of the spectrum, in terms of SNR or tCho signal amplitude, is performed within the studies using this method. To date, 13 studies [3-5,18, 23-31] using this approach to breast lesion evaluation have been published. All of these investigations were performed using $1.5 \mathrm{~T}$ machines. A summary of the reported qualitative literature is documented in Table 1.

Analysis by Katz-Brull et al. [11] of three of the qualitative studies $[3,24,25]$ examined the sensitivity of cancer diagnosis with regard to lesion size. The study data were stratified into three groups by the size of the examined lesion $(<2.5,2.5$ to 4.9 , and $\geq 5 \mathrm{~cm})$. It was noted that the sensitivity of qualitative tCho detection, and hence cancer diagnosis, significantly increased with increasing lesion size from $72 \%$ to $90 \%$ to $100 \%$ $(P=0.025)$. Hence, smaller cancers have a greater tendency to be diagnosed as benign due to insufficient tCho signal for detection.

In order to improve the sensitivity for qualitatively detecting smaller cancers, and likewise with the utility of the quantitative methods discussed later, it is necessary to increase the SNR. In order to increase the tCho signal, the use of clinical MR machines with field strengths of greater than $1.5 \mathrm{~T}$, which have increasing installation in centres worldwide, could be used. The $20034 \mathrm{~T}$ study by Bolan et al. [32] was the first to report the technique at higher field strength. In terms of additional hardware factors, innovations in specialised MR breast coil design and sensitivity might also improve the detectability of tCho. With regard to ${ }^{1} \mathrm{H}$-MRS parameters, the signals within the chemical shift range of tCho can be detected with greater sensitivity using optimised MR pulse sequences.

\section{Quantitative approaches to tCho}

Several different quantitative approaches to tCho processing have been reported. Compared to qualitative approaches, quantitative results can be more objectively compared between different sites and systems, allow for stronger statistical analyses, and can be used to measure change in longitudinal studies, such as response to chemotherapy. There is only one study investigating tCho amplitudinal scan-rescan reproducibility, reporting nonsignificant variability, though no publications to date have reported multi-site consistency for any of the qualitative or quantitative approaches [27]. There is a great need to assess the reproducibility of the modality, especially with regard to the absolute values of choline concentration obtained using the quantitative approaches. Quantitative approaches using the tCho peak integral, SNR and referencing methods are discussed below.

\section{tCho peak integral}

A 2009 1.5 T SV study by Sardanelli et al. [33] reported the use of the tCho peak integral for discriminating malignancy from benign tissue. Using spectra from 45 lesions, $89.5 \%$ sensitivity and $92.3 \%$ specificity were attained for the differentiation of malignant from benign breast lesions. Whilst this approach demonstrates an impressive level of diagnostic accuracy, the authors note that, due to the expression of the tCho peak interval in terms of arbitrary units, it may not be possible to use the reported thresholds in differing technical and clinical situations.

\section{tCho signal-to-noise ratio approach}

This methodology involves the determination of the SNR of the spectral region about which the tCho peak would be expected (approximately $3.2 \mathrm{ppm}$ ). This entails firstly quantifying the tCho peak signal amplitude, then sampling a region of the spectrum at which no signal would be expected (for example, $>6$ or $<0 \mathrm{ppm}$ ) to determine noise intensity. If the resulting SNR is higher than a study defined cutoff value (for example, $S N R \geq 2$ ), 
Table 1. Summary of qualitative ${ }^{1} \mathrm{H}-\mathrm{MRS}$ literature published to date

\begin{tabular}{|c|c|c|c|c|c|}
\hline Study & $n$ & Technique & Sensitivity & Specificity & PPV \\
\hline Roebuck et al. 1998 [3] & 17 & SV & $70 \%$ & $86 \%$ & $88 \%$ \\
\hline Gribbestad et al. 1998 [23] & 22 & SV & $50 \%$ & $100 \%$ & $100 \%$ \\
\hline Kvistad et al. 1999 [4] & 40 & SV & $82 \%$ & $61 \%$ & $82 \%$ \\
\hline Cecil et al. 2001 [24] & 38 & SV & $83 \%$ & $87 \%$ & $90 \%$ \\
\hline Yeung et al. 2001 [25] & 30 & SV & $92 \%$ & $83 \%$ & $97 \%$ \\
\hline Jagannathan et al. 2001 [5] & 46 & SV & $81 \%$ & $86 \%$ & $93 \%$ \\
\hline Kim et al. 2003 [26] & 35 & SV & $100 \%$ & $100 \%$ & $100 \%$ \\
\hline Joe et al. 2005 [27] & 15 & SV & $100 \%$ & NA & NA \\
\hline Stanwell et al. 2005 [28] & 64 & SV & $80 \%$ & $86 \%$ & $84 \%$ \\
\hline Jacobs et al. 2005 [18] & 9 & MRSI & $80 \%$ & $100 \%$ & $100 \%$ \\
\hline Geraghty et al. 2008 [29] & 16 & SV & $88 \%$ & NA & NA \\
\hline Baltzer et al. 2011 [30] & 62 & SV & $62 \%$ & $86 \%$ & $90 \%$ \\
\hline Chen et al. 2011 [31] & 62 & SV & $73 \%$ & NA & NA \\
\hline
\end{tabular}

$n=$ number of participants. 'H-MRS, magnetic resonance spectroscopy; MRSI, magnetic resonance spectroscopic imaging; NA, not applicable; PPV, positive predictive value; SV, single-voxel.

then the resonance at approximately $3.2 \mathrm{ppm}$ is assumed to correspond to tCho.

The qualitative and SNR techniques are both based upon the premise that if sufficiently large concentrations of choline-containing metabolites are present within a given voxel of interest (VOI), then a tCho peak is more likely to be observed/detectable. However, several important issues confound this assumption, since the sensitivity to detect tCho signals vary, depending on the following factors. The sensitivity of MR signal detection is directly proportional to the volume of the VOI and approximately proportional to increasing magnetic field strength $\left(\mathrm{B}_{0}\right)$. SNR also depends upon the specific breast coil design used (widely variable according to model), the VOI positioning in relation to the breast coil elements, and the variable coil loading resulting from diverse patient habitus [34].

Distinct from the sensitivity of detection, several other factors also influence the acquired signal intensity of a tCho resonance, including the intrinsic $\mathrm{T} 1$ and $\mathrm{T} 2$ relaxation times of the various chemical components contributing to the resonance, the sequence repetition time (TR) and echo time (TE) values, the capability of shimming to optimise the homogeneity of $\mathrm{B}_{0}$ over the VOI, as well as acquisition parameters such as spectral width and receiver gain.

Nine studies investigating the SNR method in breast cancer assessment have been published to date. All of these investigations were performed using $1.5 \mathrm{~T}$ machines. Table 2 summarises the reported SNR method literature.

\section{Externally phantom referenced tCho approach}

The external phantom reference method (EPRM) involves the quantification of tCho, to produce a molar concentration value, based upon the ratio of the tCho signal amplitude acquired from an in vivo VOI to that from a solution of known phosphocholine concentration within a 'phantom' container positioned outside the patient's body. The advantages of this method compared to the use of an internal reference such as endogenous water or lipid are with regard to it being a more reliable referencing method with less of the operator dependence (VOI positioning to avoid inclusion of adipose tissue) and physiologic dependence (tissue water content can vary with the menstrual cycle) [35] inherent to internal water referencing. One disadvantage of this approach is the additional time required to perform ${ }^{1} \mathrm{H}$-MRS separately on the external standard phantom (eight minutes in the Bakken study) [36] with clear repercussions on patient throughput and cost-effectiveness. Additionally, this method may require the acquisition of a sensitivity map, which also increases the time required.

To date, two studies have reported use of the EPRM in the evaluation of breast cancer in vivo. The first study to look at the quantification of tCho in breast cancer in this manner was performed in 1998 at $1.5 \mathrm{~T}$ by Roebuck et al. [3]. This group used an external phantom quantification strategy, which involved performing ${ }^{1} \mathrm{H}-\mathrm{MRS}$ on a phantom solution of known choline concentration $(1 \mathrm{mmol} / \mathrm{L})$ following the in vivo spectroscopic evaluation of the lesion of interest. The tCho SNR was then calculated both in vivo and in the phantom solution. The concentration of choline in each VOI was determined after voxel volume and full width at half maximum (FWHM) values were adjusted for. A quantifiable tCho peak was detected in 7 out of 10 malignancies, with the in vivo choline concentration ([Cho]) ranging from 0.7 to 
Table 2. Signal-to-noise ratio approach results published to date

\begin{tabular}{|c|c|c|c|c|c|}
\hline Study & $n$ & Type & tCho SNR method & Sensitivity & Specificity \\
\hline Tse et al. 2003 [50] & 40 & SV & $\geq 2$ malignant & $89 \%$ & $100 \%$ \\
\hline Huang et al. 2004 [6] & 50 & SV & $\geq 2$ malignant & $100 \%$ & $67 \%$ \\
\hline Jacobs et al. 2004 [17] & 15 & MRSI & $\geq 5$ malignant & $87 \%$ & $85 \%$ \\
\hline Bartella et al. 2006 [51] & 57 & SV & $\geq 2$ malignant & $100 \%$ & $88 \%$ \\
\hline Bartella et al. 2007 [52] & 32 & SV & $\geq 2$ malignant & $100 \%$ & $85 \%$ \\
\hline Baek et al. 2008 [8] & 36 & MRSI & $>3.2$ malignant & $81 \%$ & $78 \%$ \\
\hline Hu et al. 2008 [15] & 9 & MRSI & $>3$ malignant & $100 \%$ & $100 \%$ \\
\hline Tozaki et al. 2009 [53] & 171 & SV & $\geq 2$ malignant & $44 \%$ & $85 \%$ \\
\hline Danishad et al. 2010 [20] & 25 & MRSI & $\geq 2$ malignant & $100 \%$ & NA \\
\hline Bathen et al. 2011 [54] & 40 & SV & $\geq 2$ malignant & $60 \%$ & NA \\
\hline
\end{tabular}

$n=$ number of participants. MRSI, magnetic resonance spectroscopic imaging; NA, not applicable; SV, single-voxel; tCho, total choline-containing resonance.

$2.1 \mathrm{mM}$ using VOI volumes ranging from 1 to $1.8 \mathrm{ml}$ in the cancerous lesions. However, a tCho resonance was also detected in a patient with a rare benign lesion, tubular adenoma. This particular lesion demonstrated a [Cho] of $5.8 \mathrm{mM}$ from a $9.8 \mathrm{ml}$ VOI.

A second group employed the EPRM at $1.5 \mathrm{~T}$ in a single patient with known breast carcinoma [36]. Table 3 summarises the reported EPRM literature.

\section{Internal water referenced tCho approach}

A recent approach in quantitative breast ${ }^{1} \mathrm{H}-\mathrm{MRS}$ first reported by Bolan et al. [32] involves the use of the nonsuppressed water resonance from the same in vivo VOI used for the suppressed tCho detection acquisition. This technique shall further be referred to as the internal water reference method (IWRM). Variations of this internal referencing approach are performed routinely in the United States for brain ${ }^{1} \mathrm{H}-\mathrm{MRS}$ studies. When implementing the IWRM, the [Cho] is calculated by means of a ratio of the tCho amplitude to the unsuppressed water amplitude. This ratio can be interpreted directly as a molal quantity (moles per mass of water), or can be converted to a molar concentration (moles per volume) by assuming a water density. Adjustments are made for differences in receiver gain settings, the number of signal averages (NSA), and MR signal relaxation rates. A large NSA is required for the water and lipid suppressed ${ }^{1} \mathrm{H}$-MRS sequence acquiring the tCho resonance due to the need to resolve the relatively low amplitude tCho resonance from the baseline noise amplitude. A low NSA is required for the ${ }^{1} \mathrm{H}-\mathrm{MRS}$ sequence acquiring the unsuppressed water spectrum due to the dominating signal amplitude of water, which is normally resolvable from the baseline noise with a single excitation.

The IWRM gives slightly higher but relatively similar [Cho] results compared with the EPRM. One of the
Table 3. In vivo external phantom reference method breast cancer literature published to date

\begin{tabular}{llc}
\hline Study & $\boldsymbol{n}$ & Breast cancer [Cho] range \\
\hline Roebuck et al. 1998 [3] & 7 & $0.4-2.1 \mathrm{mmol} / \mathrm{Kg}$ \\
Bakken et al. 2001 [36] & 1 & $2.0 \mathrm{mmol} / \mathrm{Kg}$ \\
\hline
\end{tabular}

$n=$ number of participants with breast cancer contributing to reported choline concentration ([Cho]) range.

benefits of the IWRM is that it is more straightforward to implement, with no VOI repositioning required and all ${ }^{1} \mathrm{H}$-MRS acquisitions being carried out during the patient examination protocol. It also intrinsically adjusts for radiofrequency transmission efficiency and radiofrequency receive sensitivity, in addition to VOI size, $\mathrm{B}_{0}$ shim effects, and the partial volume effect resulting from the inclusion of adipose tissue [37].

The IWRM has been used to investigate cancerous breast lesions in seven studies at a range of clinical grade field strengths. A summary of the IWRM literature is detailed in Table 4.

\section{Breast lesion diagnosis when combining MRI and SV ${ }^{1} \mathrm{H}-\mathrm{MRS}$}

In 2005 Meisamy et al. [38] reported a four reader blinded observer performance study $(n=55)$ performed at $4 \mathrm{~T}$ evaluating the addition of the IWRM to DCE-MRI for breast lesion assessment. The sensitivity and specificity of diagnosis using tCho concentration alone, with a cutoff threshold of $1.05 \mathrm{mmol} / \mathrm{kg}$ (as determined by receiver operating characteristic (ROC) curve analysis) were $69 \%$ and $90 \%$, respectively. When the DCE-MRI and ${ }^{1} \mathrm{H}$-MRS findings were used in conjunction, the sensitivity and specificity were reported to be $94 \%$ and $57 \%$, respectively. The sensitivity and specificity of DCE-MRI increased from $87 \%$ to $94 \%$ and $51 \%$ to $57 \%$, respectively, once ${ }^{1} \mathrm{H}$-MRS results were accounted for. 
Table 4. In vivo internal water reference method breast cancer literature published to date

\begin{tabular}{|c|c|c|c|c|c|c|}
\hline Study & $\mathrm{B}_{0}$ & $n$ & Method & Breast cancer [Cho] range & Sensitivity & Specificity \\
\hline Bolan et al. 2003 [32] & $4 \mathrm{~T}$ & 28 & SV & $0.5-8.6 \mathrm{mmol} / \mathrm{kg}$ & $46 \%$ & $94 \%$ \\
\hline Baik et al. 2006 [55] & $1.5 \mathrm{~T}$ & 32 & SV & $0.76-21.20 \mathrm{mmol} / \mathrm{kg}$ & NA & NA \\
\hline Baek et al. 2008 [56] & $1.5 \mathrm{~T}$ & 32 & SV & $0.32-10.47 \mathrm{mmol} / \mathrm{kg}$ & NA & NA \\
\hline Sijens et al. 2010 [21] & $1.5 \mathrm{~T}$ & 2 & MRSI & 4.1 and $4.6 \mathrm{mmol} / \mathrm{kg}$ & NA & NA \\
\hline Chen et al. 2011 [31] & $1.5 \mathrm{~T}$ & 45 & SV & $0.08-9.99 \mathrm{mmol} / \mathrm{kg}$ & NA & NA \\
\hline Dorrius et al. 2011 [22] & $1.5 \mathrm{~T}$ & 24 & MRSI & $1.7-11.8 \mathrm{mmol} / \mathrm{kg}$ & $100 \%$ & $100 \%$ \\
\hline Thakur et al. 2011 [57] & $1.5 \mathrm{~T}$ & 57 & SV & $0-47.1 \mathrm{mmol} / \mathrm{kg}$ & $75-96 \%{ }^{\mathrm{a}}$ & $93-100 \%^{\mathrm{a}}$ \\
\hline
\end{tabular}

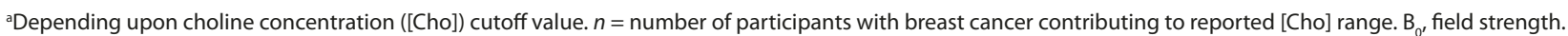
MRSI, magnetic resonance spectroscopic imaging; NA, not applicable; SV, single-voxel; T, Tesla.

The 2004 SNR paper by Huang et al. [6] also reported the sensitivity and specificity of breast cancer detection when DCE-MRI, SNR method SV ${ }^{1} \mathrm{H}-\mathrm{MRS}$ and $\mathrm{T}_{2}{ }^{*}$ weighted perfusion MR imaging results were combined within the examination. DCE-MRI alone demonstrated $100 \%$ sensitivity and $62.5 \%$ specificity. Specificity improved to $87.5 \%$ when ${ }^{1} \mathrm{H}$-MRS findings were integrated and increased further to $100 \%$ once perfusion MR results were considered. This clearly highlights the benefit of incorporating secondary MR modalities into the routine breast MR examination with regard to increasing specificity in breast lesion diagnosis.

\section{${ }^{1} \mathrm{H}-\mathrm{MRS}$ in assessing tumour responsiveness to chemotherapy}

Neoadjuvant chemotherapy (NACT), consisting of systemic agents provided preoperatively, increases the likelihood of breast conserving surgery in lieu of mastectomy when used in a patient with a chemoresponsive tumour. A therapeutic response associated with a reduction in tumour size may permit downstaging of the disease and consequently enable excision of what previously were inoperable lesions. In addition, this mode of treatment enables the tumour response to be monitored in vivo. The total disappearance of tumour at the time of resection is associated with the most favourable overall survival [39].

Given the wide range of response to chemotherapeutic agents indicated for breast cancer, it is useful to identify the effectiveness of a particular regimen at an early stage in the treatment. The methods used to determine breast cancer responsiveness to NACT can give variable results and, at present, the traditional methods of clinical examination, mammography and ultrasound, are used routinely in the clinic. Clinical MRI is playing an increasing role in the evaluation of invasive breast cancer, particularly in the trial setting.

With respect to NACT monitoring, there is a correlation between clinical response and changes in MRI-derived characteristics such as lesion size and dynamic contrast enhancement [40,41]. Unfortunately, however, such alterations cannot be identified until several weeks into a regimen, thus inhibiting the ability to optimally tailor treatment to patients on the basis of demonstrable antitumour activity $[42,43]$.

As alluded to, the capability to determine tumour responsiveness to a particular chemotherapeutic regimen in a timely manner would be highly preferable, enabling superior tailoring of a NACT regimen with the objective of achieving a full pathological response. The use of ${ }^{1} \mathrm{H}$ MRS for this purpose has been the subject of relatively small-scale investigation to date.

The first study to report a change of the tCho signal in breast cancer in response to NACT was conducted in 1999 by Kvistad et al. [4] at $1.5 \mathrm{~T}$. The qualitative SV methodology used demonstrated that a single patient with an observable tCho resonance from their invasive ductal carcinoma at pre-treatment baseline ${ }^{1} \mathrm{H}$-MRS examination no longer had a detectable tCho signal following that regimen. This finding was accompanied by a clinical response marked by a significant reduction in tumour diameter (5.7 to $3.7 \mathrm{~cm}$ ) as determined using DCE-MRI.

The next study, by Jagannathan et al. [5] at $1.5 \mathrm{~T}$ in 2001, reported sequential qualitative SV ${ }^{1} \mathrm{H}$-MRS findings both at pre-treatment baseline and within the first NACT regimen. Ten patients demonstrated an observable tCho at pre-treatment baseline, but subsequently had either absent or significantly reduced tCho one week into the regimen. These spectral findings correlated with both a clinical and histopathological response in seven of these ten cases. Both of these initial studies suggest that the qualitative assessment of tCho may have a role in monitoring the chemoresponsiveness of a tumour to a NACT regimen.

The first SV ${ }^{1} \mathrm{H}-\mathrm{MRS}$ study to investigate the quantification of tCho as a biomarker of therapeutic response to NACT was performed using the IWRM at $4 \mathrm{~T}$ in 2004 by Meisamy et al. [44]. The small cohort involved, comprising 13 participants, consisted of women 
with breast cancer scheduled to undergo a doxorubicin (anthracycline)-based NACT regimen. The difference in [Cho] from pre-treatment baseline to one day following the initial doxorubicin administration demonstrated a statistically significant $(P=0.001)$ positive correlation with the difference in tumour size after four treatment cycles compared to baseline.

A 2009 1.5 T SV IWRM study by Baek et al. [45] assessed 35 breast cancer patients from pre-NACT baseline to the first follow-up assessment following one to two cycles of doxorubicin and cyclophosphamide (DC) and a second assessment following either two further cycles of DC or a taxane-based regimen. They reported that, at second follow-up, patients who went on to have a pathologically complete response were more likely to demonstrate a significantly greater reduction in tCho concentration relative to the change in tumour size than those who had an incomplete response.

A small-scale 20101.5 T SV EPRM study by Tozaki et al. [46] assessing the response of 16 patients with breast cancer after two cycles of anthracycline-based NACT reported that there was a statistically significant difference $(P=0.004)$ in normalised choline signal between pathological responders and non-responders. A 2008 in vivo EPRM study by the same group [47] compared the ability of the integral tCho signal to evaluate early responsiveness of breast cancer to NACT compared with use of the standardised uptake value with ${ }^{18} \mathrm{~F}$-fluorodeoxyglucose positron emission tomography (PET). They reported that ${ }^{1} \mathrm{H}-\mathrm{MRS}$ is significantly correlated with the peak standardised uptake value both during and following NACT cycles $(P=0.03$ and $P<0.001$, respectively), demonstrating that ${ }^{1} \mathrm{H}-\mathrm{MRS}$ may represent a viable alternative modality to sequential PET examinations for this purpose.

The first study to investigate the use of MRSI in assessing chemoresponsiveness was reported in 2010 by Danishad et al. [20]. The SNR approach was implemented in 25 patients at $1.5 \mathrm{~T}$. Using ROC analysis, they reported that a baseline to post-third NACT cycle reduction in tCho SNR threshold of 53\% showed a sensitivity and specificity of $85.7 \%$ and $91 \%$, respectively, for differentiating clinical responders from non-responders. Clinical response was represented by a $50 \%$ reduction in tumour volume (baseline to post-third NACT cycle), as assessed by lesion size on palpation. The mean reduction in tCho SNR following one cycle of NACT was $27.8 \pm 7.8 \%$ for clinical responders and $8.6 \pm 3.9 \%$ for non-responders.

Such preliminary results highlight a promising method by which to approximate the eventual clinical response of a tumour to NACT. Larger-scale trials are necessary in order to further evaluate the utility of ${ }^{1} \mathrm{H}$-MRS for this purpose. Results from the recently completed North American multicentre ACRIN 6657 trial, which used
${ }^{1} \mathrm{H}-\mathrm{MRS}$ and DCE-MRI to assess NACT response, are still pending.

\section{Impact of gadolinium-based contrast agents on ${ }^{1} \mathbf{H}-\mathbf{M R S}$}

The majority of previous in vivo ${ }^{1} \mathrm{H}$-MRS studies involved the identification and localisation of one or more enhancing lesions based upon post-contrast imaging. However, this approach assumes the impact of gadolinium-based contrast agents on spectroscopic results is negligible.

A 2009 study performed at $3 \mathrm{~T}$ by Lenkinski et al. [48] investigated the spectroscopic effect of six commercial gadolinium-based contrast agents (Dotarem ${ }^{\circ}$, Magnevist ${ }^{\circ}$, MultiHance ${ }^{\odot}$, Omniscan ${ }^{\odot}$, OptiMark ${ }^{\odot}$ and ProHance ${ }^{\odot}$ ), all of which are currently approved for intravenous human use during MR examinations in the UK. This study examined the impact each of these agents had on the tCho peak using both phantom and rat cancer models.

Three of these agents (Omniscan ${ }^{\odot}$, OptiMark ${ }^{\ominus}$ and ProHance ${ }^{\odot}$ possess an overall neutral charge under physiological $\mathrm{pH}$ conditions, whilst the other three (Magnevist $^{\odot}$, MultiHance ${ }^{\odot}$ and Dotarem ${ }^{\odot}$ ) have a net negative (ionic) charge. Only the three ionic agents were found to bind, in an ionic manner, with choline. The interaction of these three particular agents with choline resulted in a mean reduction in the in vivo spectral tCho peak area of $40 \%$, whilst the neutral agents showed minimal or no effect. There was a statistically significant difference in the average decrease in tCho peak area between the two agent groups in vivo $(P<0.001)$. The use of the aforementioned negatively charged agents might therefore result in underestimated choline levels in patients with underlying breast cancer. On this basis, the authors went on to recommend only the administration of neutral agents for breast MR examinations involving post-contrast image localised ${ }^{1} \mathrm{H}$-MRS protocols.

A 2011 in vivo study by Baltzer et al. [30] compared ionic (Gd-DTPA; Magnevist ${ }^{\circ}$ ) and neutral (Gd DTPABMA; Omniscan ${ }^{\odot}$ ) contrasts, each at a concentration of $0.1 \mathrm{mmol} / \mathrm{kg}$ of bodyweight. All ${ }^{1} \mathrm{H}$-MRS sequences were performed 10 to 12 minutes post-administration. Significantly (45\%) lower tCho signal intensity was reported in women who were administered ionic contrast, reflecting an agreement with the Lenkinski et al. breast cancerbearing rat results reporting a $40 \%$ decrease in tCho signal. No increase in false negative rates with qualitative ${ }^{1} \mathrm{H}$-MRS was reported; however, it is important to emphasise the significance of this contrast agent charge related bias with regard to the implementation of quantitative lesion assessment and inter-study comparisons.

A 2005 in vivo study by Joe et al. [27] reported disadvantageous effects of the neutral charge Omniscan ${ }^{\circ}$ on breast cancer lesion tCho resonance when ${ }^{1} \mathrm{H}$-MRS 
was performed pre- and post-contrast. There was a significant $(P<0.0001)$ decrease in tCho amplitude 15 minutes following a $20 \mathrm{ml}$ bolus of Omniscan ${ }^{\circ}$, with an average decrease of $20 \%$ (range 3.3 to $52 \%$ ). This study also reported non-significant variations in tCho amplitude from examinations performed on separate days over a seven-day period, suggesting a greater impact of contrast on tCho amplitude compared to the variability of repeated measurements.

Given that usage of certain gadolinium-based contrast agents can be associated with a reduced tCho peak, there could be the potential for failing to detect malignancy or overestimating tumour responsiveness to NACT. A 2011 $3 \mathrm{~T}$ study by Kawai et al. [49] investigated whether the SV ${ }^{1} \mathrm{H}$-MRS VOI could be positioned accurately on breast lesions before gadolinium-based contrast agent administration, using a combination of diffusion weighted imaging (DWI) and fat suppressed T2 weighted imaging (T2WI). Using DCE-MRI performed later in the same MR session as the reference, the VOI was positioned entirely within the target lesion using DWI/T2WI in 64 (65\%) of 98 lesions. This group also compared the tCho resonances from the breast cancer lesions both before and after administration of Magnevist ${ }^{\circ}$ (gadopentetate dimeglumine) in 44 of the patients with good precontrast VOI positioning. They reported that the integral of the tCho peak was significantly greater in the precontrast versus post-contrast ${ }^{1} \mathrm{H}-\mathrm{MRS}$ acquisitions $(P<0.001)$.

\section{Conclusion}

${ }^{1} \mathrm{H}$-MRS using the tCho biomarker can provide clinically beneficial additional information in the context of breast lesion diagnosis and remains an area under active research. This adjunct modality involves the appending of at least an additional 10 minutes to the existing breast MR examination duration, with obvious repercussions on patient comfort and suitability. A growing number of higher field strength commercial clinical MR systems featuring ${ }^{1} \mathrm{H}$-MRS capabilities are being implemented. Further large-scale multi-centre and model-based research geared towards examining reproducibility is imperative. Once robustly established, the technique has the potential to increase the clinical value of the breast MR examination.

\footnotetext{
Abbreviations

${ }^{1} \mathrm{H}$-MRS, magnetic resonance spectroscopy; $\mathrm{B}_{0^{\prime}}$ main constant magnetic field of MRI machine; [Cho], choline concentration; DCE, dynamic contrast enhanced; EPRM, external phantom reference method; IWRM, internal water reference method; $M R$, magnetic resonance; $M R I$, magnetic resonance imaging; MRSI, magnetic resonance spectroscopic imaging; NACT, neoadjuvant chemotherapy; NSA, number of signal averages; ppm, parts per million; $\mathrm{PET}$, positron emission tomography; $\mathrm{ROC}$, receiver operating characteristic; SNR, signal-to-noise ratio; SV, single-voxel; T, Tesla; tCho, total choline-containing resonance; $\mathrm{VOI}$, voxel of interest.
}

Competing interests

The authors declare that they have no competing interests.

\section{Acknowledgement}

Dr Bolan wishes to acknowledge support from the NIH for the P41-RR008079 grant.

\section{Author details}

${ }^{1}$ School of Medicine and Dentistry, University of Aberdeen, Foresterhill, Aberdeen AB25 2ZD, Scotland, UK. ${ }^{2}$ Aberdeen Biomedical Imaging Centre, Medical School, Foresterhill, Aberdeen AB25 2ZD, Scotland, UK. ${ }^{3}$ Center for Magnetic Resonance Research, Department of Radiology, University of Minnesota, Minneapolis, MN 55455, USA. ${ }^{4}$ University of Cambridge, Radiology Department, Addenbrooke's Hospital, Cambridge CB2 0QQ, UK.

Published: 19 April 2012

\section{References}

1. Jemal A, Siegel R, Xu J, Ward E: Cancer statistics 2010. CA Cancer J Clin 2010, 60:277-300.

2. Jagannathan NR, Singh M, Govindaraju V, Raghunathan P, Coshic O, Julka PK, Rath GK: Volume localized in vivo proton MR spectroscopy of breast carcinoma: variation of water-fat ratio in patients receiving chemotherapy. NMR Biomed 1998, 11:414-422.

3. Roebuck JR, Cecil KM, Schnall MD, Lenkinski RE: Human breast lesions: characterization with proton MR spectroscopy. Radiology 1998, 209:269-275.

4. Kvistad KA, Bakken IJ, Gribbestad IS, Ehrnholm B, Lundgren S, Fjøsne HE, Haraldseth O: Characterization of neoplastic and normal human breast tissues with in vivo 'H MR spectroscopy. J Magn Reson Imaging 1999, 10:159-164.

5. Jagannathan NR, Kumar M, Seenu V, Coshic O, Dwivedi SN, Julka PK, Srivastava A, Rath GK: Evaluation of total choline from in-vivo volume localized proton MR spectroscopy and its response to neoadjuvant chemotherapy in locally advanced breast cancer. Br J Cancer 2001, 84:1016-1022.

6. Huang W, Fisher PR, Dulaimy K, Tudorica LA, O'Hea B, Button TM: Detection of breast malignancy: diagnostic MR protocol for improved specificity. Radiology 2004, 232:585-591.

7. Meisamy S, Bolan PJ, Baker EH, Pollema MG, Le CT, Kelcz F, Lechner MC, Luikens BA, Carlson RA, Brandt KR, Amrmi KK, Nelson MT, Everson LI, Emory $\mathrm{TH}$, Tuttle TM, Yee D, Garwood M: Adding in vivo quantitative ${ }^{1} \mathrm{H}$ MR spectroscopy to improve diagnostic accuracy of breast MR imaging: preliminary results of Observer Performance Study at 4.0 T. Radiology 2005, 236:465-475.

8. Baek H, Yu HJ, Chen J, Nalcioglu O, Su M: Quantitative correlation between 'H MRS and dynamic contrast-enhanced MRI of human breast cancer. Magn Reson Imaging 2008, 26:523-531.

9. Sitter B, Sonnewald U, Spraul M, Fjösne HE, Gribbestad IS: High-resolution magic angle spinning MRS of breast cancer tissue. NMR Biomed 2002, 15:327-337.

10. Podo F: Tumour phospholipid metabolism. NMR Biomed 1999, 12:413-439.

11. Katz-Brull R, Seger D, Rivenson-Segal D, Rushkin E, Degani H: Metabolic markers of breast cancer: enhanced choline metabolism and reduced choline-ether phospholipid synthesis. Cancer Res 2002, 62:1966-1970.

12. Ting Y-LT, Sherr D, Degani H: Variations in energy and phospholipid metabolism in normal and cancer human mammary epithelial cells. Anticancer Res 1996, 16:1381-1388.

13. Aboagye EO, Bhujwalla ZM: Malignant transformation alters membrane choline phospholipid metabolism of human mammary epithelial cells. Cancer Res 1999, 59:80-84

14. Drost DJ, Riddle WR, Clarke GD: Proton magnetic resonance spectroscopy in the brain: report of AAPM MR Task Group \#9. Med Phys 2002, 29:2177-2197.

15. Hu J, Yu Y, Kou Z, Huang W, Jiang Q, Xuan Y, Li T, Sehgal V, Blake C, E Haacke M, Soulen $\mathrm{RL}$ : A high spatial resolution ${ }^{1} \mathrm{H}$ magnetic resonance spectroscopic imaging technique for breast cancer with a short echo time. Magn Reson Imaging 2008, 26:360-366.

16. Su MY, Baik HM, Yu HJ, Chen JH, Mehta RS, Nalcioglu O: Comparison of choline and pharmacokinetic parameters in breast cancer measured by MR spectroscopic imaging and dynamic contrast enhanced MRI. Technol Cancer Res Treat 2006, 5:401-410. 
17. Jacobs MA, Barker PB, Bottomley PA, Bhujwalla Z, Bluemke DA: Proton magnetic resonance spectroscopic imaging of human breast cancer: a preliminary study. J Magn Reson Imaging 2004, 19:68-75.

18. Jacobs MA, Barker PB, Argani P, Ouwerkerk R, Bhujwalla ZM, Bluemke DA: Combined dynamic contrast enhanced breast MR and proton spectroscopic imaging: a feasibility study. J Magn Reson Imaging 2005, 21:23-28.

19. Baek HM, Chen JH, Yu HJ, Mehta R, Nalcioglu O, Su MY: Detection of choline signal in human breast lesions with chemical-shift imaging. J Magn Reson Imaging 2008, 27:1114-1121.

20. Danishad KKA, Sharma U, Sah RG, Seenu V, Parshad R, Jagannathan NR: Assessment of therapeutic response of locally advanced breast cancer $(\mathrm{LABC})$ patients undergoing neoadjuvant chemotherapy (NACT) monitored using sequential magnetic resonance spectroscopic imaging (MRSI). NMR Biomed 2010, 23:233-241.

21. Sijens PE, Dorrius MD, Kappert P, Baron P, Pijnappel RM, Oudkerk M: Quantitative multivoxel proton chemical shift imaging of the breast. Magn Reson Imaging 2010, 28:314-319.

22. Dorrius MD, Pijnappel RM, Jansen-van der Weide MC, Jansen L, Kappert P, Oudkerk M, Sijens PE: Determination of choline concentration in breast lesions: quantitative multivoxel proton MR spectroscopy as a promising noninvasive assessment tool to exclude benign lesions. Radiology 2011, 259:695-703.

23. Gribbestad IS, Singstad TE, Nilsen G, Fjøsne HE, Engan T, Haugen OA, Rinck PA: In vivo ${ }^{1} \mathrm{H}$ MRS of normal breast and breast tumors using a dedicated double breast coil. J Magn Reson Imaging 1998, 8:1191-1197.

24. Cecil K, Schnall M, Siegelman E, Lenkinski R: The evaluation of human breast lesions with magnetic resonance imaging and proton magnetic resonance spectroscopy. Breast Cancer Res Treat 2001, 68:45-54.

25. Yeung DKW, Cheung HS, Tse GMK: Human breast lesions: characterization with contrast-enhanced in vivo proton MR spectroscopy-initial results. Radiology 2001, 220:40-46.

26. Kim J, Park S, Lee HM, Lee YH, Sung NK, Chung DS, Kim OD: In vivo ' ${ }^{1} \mathrm{H}-\mathrm{MRS}$ evaluation of malignant and benign breast diseases. Breast 2003, 12:179-182.

27. Joe BN, Chen VY, Salibi N, Fuangtharntip P, Hildebolt CF, Bae KT: Evaluation of ${ }^{1} \mathrm{H}$-magnetic resonance spectroscopy of breast cancer pre- and postgadolinium administration. Invest Radiol 2005, 40:405-411.

28. Stanwell P, Gluch L, Clark D, Tomanek B, Baker L, Giuffrè B, Lean C, Malycha P, Mountford C: Specificity of choline metabolites for in vivo diagnosis of breast cancer using ${ }^{1} \mathrm{H}$ MRS at 1.5 T. Eur J Radio/ 2005, 15:1037-1043.

29. Geraghty PR, van den Bosch MAAJ, Spielman DM, Hunjan S, Birdwell RL, Fong KJ, Stables LA, Zakhour M, Herfkens RJ, Ikeda DM: MRI and ${ }^{1} \mathrm{H}$ MRS of the breast: presence of a choline peak as malignancy marker is related to k21 value of the tumor in patients with invasive ductal carcinoma. Breast $J$ 2008, 14:574-580.

30. Baltzer PA, Gussew A, Dietzel M, Rzanny R, Gajda M, Camara O, Reichenbach $J R$, Kaiser WA: Effect of contrast agent on the results of in vivo ${ }^{1} \mathrm{H}$ MRS of breast tumors - is it clinically significant? NMR Biomed 2011, 25:67-74.

31. Chen JH, Mehta RS, Baek HM, Nie K, Liu H, Lin MQ, Yu HJ, Nalcioglu O, Su MY: Clinical characteristics and biomarkers of breast cancer associated with choline concentration measured by ${ }^{1}$ H MRS. NMR Biomed 2011, 24:316-324.

32. Bolan PJ, Meisamy S, Baker EH, Lin J, Emory T, Nelson M, Everson LI, Yee D, Garwood M: In vivo quantification of choline compounds in the breast with ${ }^{1} \mathrm{H}$ MR spectroscopy. Magn Reson Med 2003, 50:1134-1143.

33. Sardanelli F, Fausto A, Leo GD, Nijs RD, Vorbuchner M, Podo F: In vivo proton MR spectroscopy of the breast using the total choline peak integral as a marker of malignancy. Am J Roentgenol 2009, 192:1608-1617.

34. Redpath TW: Signal-to-noise ratio in MRI. Br J Radiol 1998, 71:704-707.

35. Graham SJ, Stanchev PL, Lloyd-Smith JO, Bronskill MJ, Plewes DB: Changes in fibroglandular volume and water content of breast tissue during the menstrual cycle observed by MR imaging at 1.5 T. J Magn Reson Imaging 1995, 5:695-701.

36. Bakken IJ, Gribbestad IS, Singstad TE, Kvistad KA: External standard method for the in vivo quantification of choline-containing compounds in breast tumors by proton MR spectroscopy at 1.5 Tesla. Magn Reson Med 2001, 46:189-192.

37. Haddadin IS, Mclntosh A, Meisamy S, Corum C, Styczynski Snyder AL, Powel NJ, Nelson MT, Yee D, Garwood M, Bolan PJ: Metabolite quantification and high-field MRS in breast cancer. NMR Biomed 2009, 22:65-76.

38. Meisamy S, Bolan PJ, Baker EH, Pollema MG, Le CT, Kelcz F, Lechner MC,
Luikens BA, Carlson RA, Brandt KR, Amrami KK, Nelson MT, Everson LI, Emory $\mathrm{TH}$, Tuttle TM, Yee D, Garwood M: Adding in vivo quantitative ${ }^{1} \mathrm{H}$ MR spectroscopy to improve diagnostic accuracy of breast MR imaging: preliminary results of Observer Performance Study at 4.0 T. Radiology 2005, 236:465-475.

39. Ogston KN, Miller ID, Payne S, Hutcheon AW, Sakar TK, Schofield A, Heys SD: A new histological grading system to assess response of breast cancers to primary chemotherapy: prognostic significance and survival. Breast 2003, 12:320-327.

40. Harry VN, Semple SI, Parkin DE, Gilbert FJ: Use of new imaging techniques to predict tumour response to therapy. Lancet Oncol 2010, 11:92-102.

41. Semple SIK, Staff RT, Heys SD, Redpath TW, Welch AE, Ahearn TS, Hutcheon AW, Gilbert FJ: Baseline MRI delivery characteristics predict change in invasive ductal breast carcinoma PET metabolism as a result of primary chemotherapy administration. Ann Oncol 2006, 17:1393-1398.

42. Rieber A, Brambs HJ, Gabelmann A, Heilmann V, Kreienberg R, Kühn T: Breast MRI for monitoring response of primary breast cancer to neo-adjuvant chemotherapy. Eur Radio/ 2002, 12:1711-1719.

43. Johansen R, Jensen LR, Rydland J, Goa PE, Kvistad KA, Bathen TF, Axelson DE, Lundgren S, Gribbestad IS: Predicting survival and early clinical response to primary chemotherapy for patients with locally advanced breast cancer using DCE-MRI. J Magn Reson Imaging 2009, 29:1300-1307.

44. Meisamy S, Bolan PJ, Baker EH, Bliss RL, Gulbahce E, Everson LI, Nelson MT, Emory TH, Tuttle TM, Yee D, Garwood M: Neoadjuvant chemotherapy of locally advanced breast cancer: predicting response with in vivo ${ }^{1} \mathrm{H} \mathrm{MR}$ spectroscopy - a pilot study at 4 T. Radiology 2004, 233:424-431.

45. Baek HM, Chen JH, Nie K, Yu HJ, Bahri S, Mehta RS, Nalcioglu O, Su MY: Predicting pathologic response to neoadjuvant chemotherapy in breast cancer by using MR imaging and quantitative ${ }^{1} \mathrm{H}$ MR spectroscopy. Radiology 2009, 251:653-662.

46. Tozaki M, Sakamoto M, Oyama Y, Maruyama K, Fukuma E: Predicting pathological response to neoadjuvant chemotherapy in breast cancer with quantitative ${ }^{1} \mathrm{H}$ MR spectroscopy using the external standard method. J Magn Reson Imaging 2010, 31:895-902.

47. Tozaki M, Sakamoto M, Oyama Y, O'uchi T, Kawano N, Suzuki T, Yamashiro N, Ozaki S, Sakamoto N, Higa K, Abe S, Ogawa T, Fukuma E: Monitoring of early response to neoadjuvant chemotherapy in breast cancer with ${ }^{1} \mathrm{H} M R$ spectroscopy: comparison to sequential 2-[18F]-fluorodeoxyglucose positron emission tomography. J Magn Reson Imaging 2008, 28:420-427.

48. Lenkinski RE, Wang X, Elian M, Goldberg SN: Interaction of gadoliniumbased MR contrast agents with choline: implications for MR spectroscopy (MRS) of the breast. Magn Reson Med 2009, 61:1286-1292.

49. Kawai H, Naganawa S, Satake H, Ishigaki S, Sakurai Y, Mori M, Maruyama K: ${ }^{1} \mathrm{H}$-magnetic resonance spectroscopy of the breast at 3.0-T: comparison of results obtained before and after administration of gadolinium-based contrast agent. J Magn Reson Imaging 2011, 35:717-722.

50. Tse GMK, Cheung HS, Pang L, Chu WCW, Law BKB, Kung FYL, Yeung DKW: Characterization of lesions of the breast with proton MR spectroscopy: comparison of carcinomas, benign lesions and phyllodes tumors. Am J Roentgenol 2003, 181:1267-1272.

51. Bartella L, Morris EA, Dershaw DD, Liberman L, Thakur SB, Moskowitz C, Guido J, Huang W: Proton MR spectroscopy with choline peak as malignancy marker improves positive predictive value for breast cancer diagnosis: preliminary study. Radiology 2006, 239:686-692.

52. Bartella L, Thakur SB, Morris EA, Dershaw DD, Huang W, Chough E, Cruz MC, Liberman L: Enhancing nonmass lesions in the breast: evaluation with proton ('H) MR spectroscopy. Radiology 2007, 245:80-87.

53. Tozaki M, Fukuma E: ${ }^{1} \mathrm{H}$ MR spectroscopy and diffusion-weighted imaging of the breast: are they useful tools for characterizing breast lesions before biopsy? Am J Roentgenol 2009, 193:840-849.

54. Bathen TF, Heldahl MG, Sitter B, Vettukattil R, Bofin A, Lundgren S, Gribbestad IS: In vivo MRS of locally advanced breast cancer: characteristics related to negative or positive choline detection and early monitoring of treatment response. MAGMA 2011, 24:347-57.

55. Baik H, Su M, Yu H, Mehta R, Nalcioglu O: Quantification of cholinecontaining compounds in malignant breast tumors by ${ }^{1} \mathrm{H} \mathrm{MR}$ spectroscopy using water as an internal reference at 1.5 T. Magn Reson Mater Phys Biol Med 2006, 19:96-104.

56. Baek HM, Chen JH, Nalcioglu O, Su MY: Proton MR spectroscopy for monitoring early treatment response of breast cancer to neo-adjuvant chemotherapy. Ann Oncol 2008, 19:1022-1024. 
57. Thakur SB, Brennan SB, Ishill NM, Morris EA, Liberman L, Dershaw DD, Bartella $L$, Koutcher JA, Huang W: Diagnostic usefulness of water-to-fat ratio and choline concentration in malignant and benign breast lesions and normal breast parenchyma: an in vivo 'H MRS study. J Magn Reson Imaging 2011, 33:855-863. doi:10.1186/bcr3132

Cite this article as: Begley JKP, et al.: In vivo proton magnetic resonance spectroscopy of breast cancer: a review of the literature. Breast Cancer Research 2012, 14:207. 